\title{
Hermeneutic Resonance in Animats and Art
}

\author{
Alasdair Turner \\ Bartlett School of Graduate Studies, UCL, Gower Street, London \\ a.turner@ucl.ac.uk
}

\begin{abstract}
One major criticism of direct or active perception (and other forms of embodied action) from the perspective of cognitive psycology is that, according to common sense, there are some actions that require strictly symbolic information - for example, to stop a car in response to a red traffic light — which fall outside the realm of a perception-action cycle. Although such cognitive responses are not necessarily a goal of artificial life, they must necessarily be included within the embodied paradigm if it is to encompass the cognisant individual, the self-aware individual, or, potentially, the conscious individual. This paper will address the question, 'can an animat appreciate art?' Although this may seem very different to the example of a prosaic response to a traffic light, it will be argued that a common framework for establishing the meaning of an object is needed. It will also be argued that clarification to previous philosophical models of artistic engagement is required: in particular that the process of understanding is not a dialogue between an autopoietic artwork and animat, but that there is either a unity of object (artworkanimat) which becomes self-maintaining, or a more classical Gibsonian interpretation as a fixed set of affordances offered by an object to the subject, both of which lead to the conclusion that the process of understanding becomes a resonance in the unity or animat.

Keywords: Autopoiesis, hermeneutics, embodiment, phenomenology, direct perception, natural vision
\end{abstract}

\section{Introduction}

The relationship between artificial life and art is an enticing one. That an artificial organism might have access to a deeper meaning within an object than is initially apparent, or, further, be able to create an object capable of engendering deeper meaning, offers an intriguing mechanical and philosophical challenge. Wheeler [1] attempts to bring together the materialism of Rothko's outlook with the spiritual experience of his art, through consideration of the philosophy of artificial life and the phenomenological relationship between art viewer and art work. In a previous study, I took Wheeler's phenomenological interpretation and applied it to the interaction between artwork and animat to create an ongoing embodied process of location and relocation of both an 'artwork' and an animat [2]. Bird and Stokes [3] are engaged in the production of fractal drawings by animats, with allusions to what surely must be the creation of art through animat. However, Bird and Stokes are careful to avoid making explicit this implication, 
as in doing so the difficult question of 'what is art?' is raised. While this question may be impossible to answer directly, this paper will attempt to show that the related question of 'what is artistic appreciation?' can be tackled through the philosophy of artificial life. Although answering this question will not determine 'what is art?', it can address questions such as 'is this specific creation artistic?'.

In wrestling with the question 'what is artistic appreciation?', it is important to realise that the analysis herein is based very much on Wheeler's account, in particular, his move to a non-linguistic hermeneutics. The symbolic representation of meaning, and Cartesian dualism are rejected in favour of a process-based approach to understanding. However, Wheeler leaves a certain amount unsaid; in his coda of 'Art and Language', there are two types of understanding process. An example of a 'live' jazz musical interaction is described as a hermeneutical dialogue, which implies a loop of (non-linguistic) information exchange between two individuals (performer and auditor), whereas the appreciation of Rothko's art is discussed in terms of a phenomenological 'drawing into the artwork', or as we will put it here, an 'at-oneness' with the art. This second mode of interaction is very much one way: the art does the drawing into, and there is little if any formal exchange of information. In [2], I took an approach which initially seems to be more fruitful: that both artwork and live jazz musician have equal status as entities, and that there is a hermeneutical dialogue in both cases. However, it now seems to me, and as will be examined, that it is the 'at-oneness' which provides for both a true account of the process of understanding. The framework constructed here also seems to lead to a measurable outcome in an animat for 'art appreciation'. If correct, this completes Wheeler's attempt to tie the scientific artificial life approach to the phenomenological account of the experience of art.

In order to get to this stage, I will draw heavily on the work of Luhmann [4]. Luhmann struggles to get to the crux of meaning within the context a systems approach, through a ten point examination of the necessary conditions for meaning. Although for the most part, Luhmann is engaged in the analysis of social systems, at this stage he maintains that 'psychic' (pertaining to the individual) and 'social' meaning are identical in form - that is, that his formal theory of meaning is equally applicable to all systems. For the purpose of this paper, we will tend to examine the individual. Wheeler takes pains to explicate the role of individual and society, as the animat apparently lacks social context, and without a social context, in Heidegger's stream of phenomenology, there can be no art. We will try to look in more detail at what the context of the animat is, both environmentally and socially, and suggest that, social context is a special case of a more general environmental context.

Hermeneutics itself is a topic too large to be covered in detail and riven with a history of linguistic approach [5]; it will be divided into two more manageable concerns: the establishment of meaning (which Luhmann will inform) and the process of understanding, which will be approached through autopoesis [6], and Gibson's affordance-based theory of vision [7]. Given the appeal to Gibson, this paper will concentrate on the discussion visual art, but other media should not 
be discounted. The generality of the approach is to sensor-motor individuals connected to the environment by any type of affordance.

Before addressing the major topic, it is worth mentioning that there exists a more prescient problem than the linguistic backdrop of hermeneutics, and that is the equation of phenomenology and science into a single framework. To the originators of hermeneutic or phenomenological enquiry the experiential cannot be resolved with the analytic, or to use Gadamer's [5] terms, truth cannot be resolved with method. However, to be true to its aims, it seems to me that the practice of artificial life should aim at this very resolution.

\section{Background}

There are various approaches to embodied $[8]$ or situated cognition $[9,10]$. At the centre of any is the rejection fundamental dualism of Descartes, and the either implicit or explicit adoption of a philosophy attuned to Heidegger's phenomenology. The basal assumption is that the organism can only exist in relationship to its environment, and that cognition can only follow in this embodied situation.

For the purposes of this paper, I will start with Maturana and Varela's definition of living existence, autopoiesis [6]. Maturana and Varela [11] draw the situation of autopoiesis, as shown in figure 1. The organism (originally in the scope of their theory, a biological cell) is drawn as a circle with an arrow, intended to indicate a self-producing entity. That is, an entity which maintains itself in relation to its environment. This environmental relationship is also shown in the diagram, with arrows to indicate that the entity is both affected by and affecting its environment.

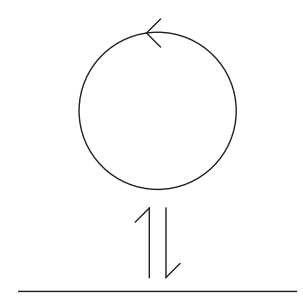

Fig. 1. Autopoietic individual in relation to the physical environment, after Maturana and Varela [11]

More formally, the autopoietic entity, a network of ongoing processes, both continuously reproduces its network of processes, and distinguishes these processes from the environment, or physical space around it. This, to proponents of autopoiesis, is life. Furthermore, this systems theory account of interaction summarises the process of direct or active perception. 
In Gibson's direct perception [7] as related to natural vision, he posits that the individual interacts directly with its environment, taking affordances from the environment in order to move around it. His affordances pre-exist in the environment, they are natural ways in which the individual can interact with the objects around it. For example, a rock might be picked up, or, to move to a Heideggerian example, a hammer may be picked up as it affords the ability to pick it up by the shape of its handle. It may also afford hammering of an object. Both of these can be seen in autopoietic terms of the individual existing in the environment, with a linkage through picking up or otherwise engaging with the object. Gibson's view is that in natural vision, if the affordance exists then it will be followed. So, a chair placed on a floor affords moving towards it, and sitting on it. An argument against Gibson is that the chair also affords standing on, being thrown across the room and various actions that although they happen, are not typical interactions with the object. Why should it be more usual to sit on a chair than to throw it? Of particular relevance, is how the object takes on the set of meanings of the chair, and how non-manipulable objects, such as fixed static artworks, take on a meaning. There is also the location of meaning to consider. In the definition of embodied cognition, there is a dilemma, in that although the dualism of Descartes (mind-body split) is rejected, there is still a distinction between entity and environment, and a fundamental distinction of meaning and non-meaning. Since the symbolism of objects has been eschewed, there has to be a replacement for the symbol.

These are issues that typically seem to be glossed over in the race for an embodied cognition. For the adherent to autopoiesis, all life might be construed as cognition [12], in that these processes of active perception simply are the processes of life. However, Luhmann, in relation to social systems, does realise the requirement for further understanding of meaning systems, and in this paper I will examine the relationship of these to cognisant systems boundaries.

\section{Meaning in Systems Theory}

Luhmann [4, pp. 59-102] grapples with 'meaning' in the context of non-symbolic systems theory in ten phases. These can be summarised as follows:

1. Luhmann discards a static approach to meaning. All meaning is dynamic, as it must be allowed to change over time and for different individuals and contexts. For our purposes, dynamical systems are well known to artificial life, and we do not need to dwell on this factor.

2. Luhmann notes that meaning must be an action of distinction, what I call above the taking on of a set of meanings. That is, where a chair becomes sittable or throwable, these meanings must be distinguishable from the previous state of non-meaning.

3. Establishing meaning necessarily involves information: the transfer of information, from information to non-information embeds meaning (where 'noninformation' is information no longer necessary to create the established meaning). 
4. Once a new meaning exists, it extends the horizon of available meanings (which Luhmann suggest's is Husserl's horizon). That is, once one meaning is established, there become other possible meanings.

5. Importantly, the subject is discarded. Luhmann asserts that meaning occurs outside of 'subject'. This has implications for boundaries of knowledge and cognition in the current context, which are dealt with in the next section.

6. Luhmann distinguishes between actuality and possibility, so that meaning is decomposed into different 'dimensions': factual (or ontological), temporal, and social (or intersubjective).

7. There is an action involved in meaning (attribution as action is the reproduction of autopoietic systems) and experience (attribution as experience is the reproduction of meaning). Luhmann posits that there can be no experience without action, that is, no ego without alter. This will be a difficulty to us in the quiet contemplation of artwork. What action is involved in the appreciation of art? This will be dealt with in the final section.

8. Meaning is an evolution: it is not planned, by which we might understand that accident of experience comes to play in the establishment of meaning - knocking over a chair accidentally may show that it embodies affordances for throwing.

9. Meaning as a symbolic generalisation must include self-reference. If it does not then it fails to include the possibility of future alteration. This forms the single most important factor to us, as it is the link to a symbolic representation. Luhmann suggests that 'the concept of symbolic generalisation of meaning's self-reference replaces 'sign", which sounds complex in the extreme, but is perhaps approximated by simply a looping or recursive relationship established which equates to a sign.

10. Meaning can be present either in the form of consciousness or communication (psychic or social), or in our case, indivdiual.

Although Luhmann suggests that meaning for systems is the totality of all these factors, some have more bearing than others. Most are either implicitly or explicitly accepted within the fields of artificial life and embodied cognition. However, for the current purpose, three stand out as requiring further attention: the loss of subjectivity (5), the action to create meaning (7), and the creation of self-reference (9). If loss of subjectivity is to be tackled in an autopoietic context, then it must explain how the individual definition of itself as distinct from the environment can be resolved with a non-locational meaning. The second of these three, action, of course should not be a problem, as the very basis of the paper is in active perception. But there is a problem as the appreciation of a static artwork initially seems inactive. The perception-action cycle is seemingly broken in the relationship of artwork to animat. This will be dealt with in the final section, and will rely on the third and most difficult of Luhmann's factors, the necessity of a self-referential loop in generalisation of meaning. 


\section{Boundaries and Affordances}

There is a problem with the autopoietic approach to cognition raised by Boden [12]. She points out that, according to Maturana and Varela's own definition, the autopoietic individual must maintain its identity as separate from the environment. This conflicts with a view of situated cognition, where knowledge is distributed between the combination of individual and environment. It is also a problem to our conception, as the locus of meaning is important to the ability to cognise. The solution proposed here is to look more closely at what the maintenance of a separate identity involves. If the individual is a human being, then it can easily add what might seem inorganic objects to itself. For example, nail varnish can be painted and repainted. Although this does not immediately seem a great step, it does suggest that the individual can adapt its boundary, and that the boundary can extend to non-organic structure. This is already explicit in autopoiesis: the nail varnish is a protective layer that encases part of the individual just as a cell wall forms an outer boundary to a cell. Both are inorganic matter, and can be added to extend the individual. However, once this step has been taken, there is no longer a fixed boundary for the individual at all. The boundary may be physical in the sense that it exists in space, but it does not necessarily follow the prescribed Kantian order around it. Gibson [7] makes the interesting observation that the third dimension is a Cartesian error, and the accepted boundary of an object forms a similar error. This, of course, is clear in phenomenological thinking: Heidegger's hammer is at one with the skilled practitioner when in use, and the boundary of the unity thus extends around them 'both'. In this sense the unity exists as what Merleau-Ponty might recognise as a series of adaptive Gestalts [13], although the word Gestalt implies a forced set of results, rather than the range of possibilities that might actually exist.

In this sense of body, action in response to affordance creates new unities of object, and these come within the modes of usage we have examined. The objections to neo-Gibsonian affordance can be seen as trivial. For example, the chair can still be stood on, thrown or sat on. The action of throwing, standing on or sitting creates a new unity of object. Luhmann's symbolic generalisation, that of chair, is created through the combination of these actions and the gathering of them together into a continued loop. In this sense, the creation of meaning is consistent with the extension of the body. Symbolic features of the environment, for example a door handle, or even the mechanism for opening a puzzle box come into being through the action of their completion. So to a culture that has not seen a door handle, the very action of performing the use of the handle becomes its meaning. The puzzle box is infuriatingly present-at-hand until we work out the relationships. Action is discovery of inherent affordances in the object. Once these are learnt, they can be applied to other similar affordances in objects, thus enforcing the symbolic meaning of the object. 


\section{Societal Context}

A rapid exposition such as this must necessarily neglect much detail. One important feature of meaning for Heidegger is the societal context of meaning. Wheeler points out that since the individual animat in a setting is devoid of societal context, the possibility of a phenomenological account of the appreciation of art seems flawed. While Wheeler's resolution, to invoke fitness affordance is undoubtedly adequate, there are a couple of further points which may prove fruitful: firstly, it is important to point that just as we do not exist in absentia of society, neither does an animat. We may attempt to divorce it, but our very positioning of motors and sensors, or the representation used in our genetic algorithm, or the task chosen for it to perform encode societal values. We should not see this as a problem, but in fact a solution: this animat remains a human creation, and is inherently (and irrevocably) linked to its creator. It is a social construction, although perhaps non-social in itself, no matter how hard we try to avoid it. The second point is that the social and physical environment of an animat may be considered as a continuous relationship, in that the situation of the societal relationships and environmental relationships can be abstracted from the animat, following previous work on the definition of relationships between individuals, society and environment [14], as shown in figure 2.

(a)

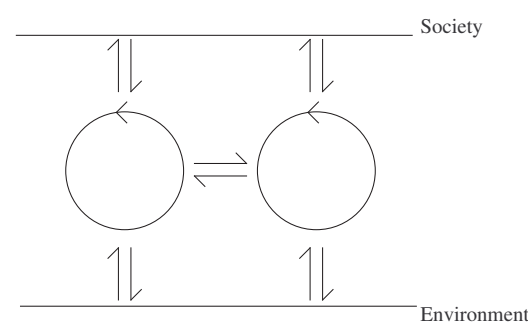

Fig. 2. (a) Autopoietic relationship between individuals, society and environment, after [14], (b) The autopoietic context of the individual.

\section{Hermeneutic Resonance}

In section 4 it was argued that the process of understanding is achieved through a creation of unity, and that this unity permits situated cognition through the combination of affordances. This is a very different model of understanding to the hermeneutical dialogue. Figure 3 shows what a hermeneutical dialogue might look like, according to my initial intepretation [2]. This was perhaps reasonable for that study, where animats and artworks both moved, creating the situation whereby an informational exchange between the two could exist; however, it 
does not serve in the situation where an artwork is seen as grounded. In this case, whatever affordance the artwork offers cannot be responded to directly. For example, how does the animat stop, look and reflect on the canvas in a meaningful exchange when the canvas offers only static affordances. In fact, of course, the canvas offers many affordances, for example, that of ripping it off the wall and constructing a dwelling with it. We must accept that this is what many cultures may do with a Rothko, but then we can invoke Luhmann's concept of a stochastic evolution of meaning to suggest that one animat might not follow this more primary affordance, it might follow another affordance offered by the painting, but what is that affordance?

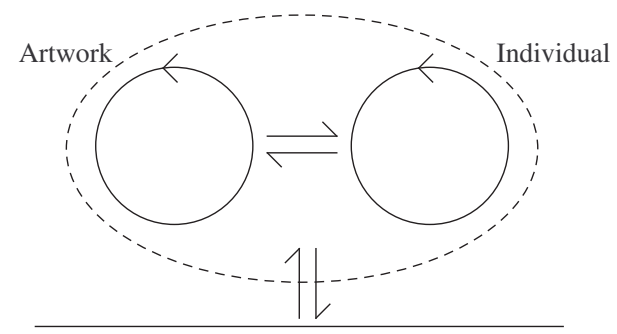

Fig. 3. Autopoietic relationship between artwork and individual, after [2]

Although the diagram in figure 3 may well show a creation of a hermeneutical unity of animat and artwork encased within the dotted border, according to Luhmann, it still falls short of the establishment of meaning, because there is no process of the creation of a self-referential generalisation of symbolism. Furthermore, as we have discussed, if the art is static, it also falls short according to Luhmann as it does not afford action in relation to the artwork. It seems to me that the resolution of this situation is one captured by figure 4 . The lefthand situation shows artwork offering affordance, without action from the animat. However, in the righthand situation, a self-referential process is established between artwork and viewer. The viewer is 'at-one' with the artwork in their contemplation of it. The affordance of the artwork is to create this very self-referential meaning loop. Thus there is an action, an intentional action, to establish the loop between the artwork, just as there is an intentional action to modify the individual by her painting her nails. In this manner, the three vital facets of Luhmann's meaning have been met: there is a non-local event concerning an action of self-referential creation.

\section{Conclusion}

In this paper, I have investigated how Luhmann's [4] work on meaning in embodied systems can be applied to the consideration of how animats might understand 


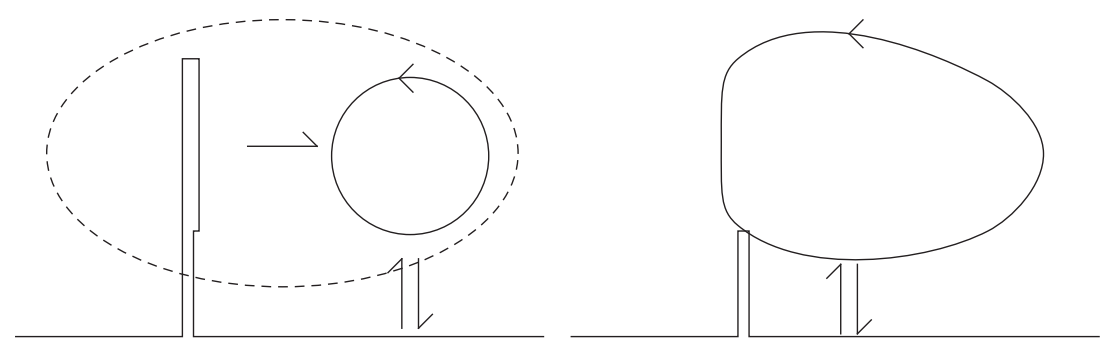

Fig. 4. Hermeneutic relationship between artwork and individual

artwork. I have argued that Wheeler's notion of a hermeneutic dialogue [1] should be extended to encompass the establishment of a self-referential unity between viewer and artwork. Although this may seem a somewhat esoteric approach, and limited in its application to artwork, the relevance to artificial life systems is that it provides a mechanism to be able to move beyond simple reactive units and into cognisant individuals without compromising on the active perception paradigm. That is, the formation of meaning cycle presented allows us to posit cognition without the requirement of a physical symbol system.

As a somewhat fanciful indulgence, it might be claimed that the formation of the cycle causes a resonance in the animat-artwork unity. But fanciful as it might be, it tallies with the phenomenological perspective of the interaction with artwork: it feels as though it causes a resonance when it transmits meaning or truth. The disengagement from the cycle leaves the animat modified, although the artwork itself remains intact from which others in society can take their own meaning. The fanciful interpretation may even extend to what an appreciation of art might look like in an animat. As a materialist, it seems to me that we should investigate the neural response of an animat in this situation: one that appears to stop and watch. Has a new resonance been created in its neurology, and has it, just possibly, appreciated art?

\section{References}

1. Wheeler, M.: From robots to Rothko: The bringing forth of worlds. In Boden, M.A., ed.: The Philosophy of Artificial Life. Oxford University Press, Oxford, UK (1996) 209-236

2. Turner, A.: Reversing the process of living: Generating ecomorphic environments. In Hanson, J., ed.: Proceedings of the 4th International Symposium on Space Syntax, London, UK, UCL (2003) 15.1-15.12

3. Bird, J., Stokes, D.: Evolving fractal drawings. In Soddu, C., ed.: Generative Art 2006, Milan, Italy (2006)

4. Luhmann, N.: Social Systems. Standford University Press, Stanford, CA (1984)

5. Gadamer, H.G.: Truth and Method. Continuum Impacts, London (2004) 
6. Maturana, H.R., Varela, F.J.: Autopoiesis and Cognition : the Realization of the Living. D. Reidel, London, UK (1980)

7. Gibson, J.J.: The Ecological Approach to Visual Perception. Houghton Mifflin, Boston, MA (1979)

8. Varela, F.J., Thompson, E., Rosch, E.: The Embodied Mind: Cognitive Science and Human Experience. MIT Press, Cambridge, MA (1993)

9. Husbands, P., Harvey, I., Cliff, D.: An evolutionary approach to situated artificial intelligence. In Sloman, A., et al., eds.: Prospects for Artificial Intelligence. IOS Press, Amsterdam, Netherlands (1993)

10. Clancy, W.J.: Situated Cognition. Cambridge University Press, Cambridge, UK (1997)

11. Maturana, H.R., Varela, F.J.: The Tree of Knowledge: The Biological Roots of Human Understanding. Shambhala Publications, Boston, MA (1987)

12. Boden, M.A.: Autopoiesis and life. Cognitive Science Quarterly 1 (2000) 117-145

13. Merleau-Ponty, M.: Phenomenology of Perception. Routledge Classics, London (2002)

14. Turner, A.: Analysing the visual dynamics of spatial morphology. Environment and Planning B: Planning and Design 30 (2003) 657-676 